\title{
Effectiveness of Two Self-Etchings Bonded Clinically in Caries Affected Dentin with Homogeneous Smear Layer
}

\author{
Roberto Pinna, Paolo Usai, Mariantonietta Arrica, and Egle Milia \\ Department of Biomedical Sciences, University of Sassari, Viale San Pietro 43/c, 07100 Sassari, Italy \\ Correspondence should be addressed to Egle Milia; emilia@uniss.it
}

Received 22 August 2014; Accepted 5 November 2014

Academic Editor: Jen-Jie Chieh

Copyright ( 2015 Roberto Pinna et al. This is an open access article distributed under the Creative Commons Attribution License, which permits unrestricted use, distribution, and reproduction in any medium, provided the original work is properly cited.

\begin{abstract}
Aim. To examine the interface of two self-etchings in carious affected dentin (CAD), under clinical conditions. Materials and Methods. Class I cavities were prepared in 21 carious premolars, refining them by the use of a fine diamond bur in order to create similar smear layer interference. The mild self-etching Clearfil SE Bond (CSE), pH = 2.0, and the strong Tyrian SPE-One Step Plus (TSO), $\mathrm{pH}=0.5$, were applied followed by a composite. Teeth were extracted and processed for TEM. The primer's osmolarity of the self-etchings was also calculated using a microosmometer. Results. CSE hybrid layer retained smear layer residues and scattered crystallites as an effect of the mild acidity. A high presence of hydroxyapatite still protected the affected fibres within the hybrid layer. CSE primer demonstrated values of $3220 \mathrm{mOsm} / \mathrm{kg}$. TSO interdiffusion showed strong demineralization. Resin tags were not formed in the dentinal tubules which remained obstructed by crystals. However, dissolution of peritubular dentin and porosities were observed in the intertubular regions. The osmolarity of TSO was $8200 \mathrm{mOsm} / \mathrm{kg}$. Conclusion. Increasing the acidity and osmolarity of the self-etchings increases interference in the homogeneous reinforcement of CAD, which may predispose the hybrid layer to instability and hydrolytic degradation overtime.
\end{abstract}

\section{Introduction}

In adhesive dentistry, carious affected dentin (CAD) represents a very common substrate. This is a result of "minimal invasion therapy" [1], which tends to fulfil the main requirements of a more conservative and aesthetic treatment with maximum conservation of tooth structure, already compromised by the bacterial infection.

Although CAD is able to interact with adhesive monomers [2], the hybrid layer may become unstable over time due to the complex tissue changes by the bacterial attack that lower the tensile strength and Knoop hardness in respect to normal dentin $[3,4]$. This has been linked to the loss of minerals in intertubular dentin [5] that, at the same time, increases wetness with interference to infiltration of the monomers $[6,7]$.

When self-etching systems are used, bond strength has been related to the homogeneity of interdiffusion proceeding from the top smear layer toward the underlying features of the affected tissue $[8,9]$. From this point of view, the maintenance of acid resistant mineral occlusions in the affected tubules [4] with prevention of resin tags is not an important reason for lower strength than sound dentin, but a nonhomogeneous, poor infiltration of CAD would be the main reason [10].

Poor infiltration may be caused by the incapacity of self-etchings to completely infiltrate a thick and irregular smear layer, particularly when mild self-etching adhesives $(\mathrm{pH}=2.0)$ are used [8]. This interference may cause a significant decrease in effectiveness of the hybrid layer after water exposure, with nanoleakage and degradation [11]. By lowering the $\mathrm{pH}$ of the primer by using strong self-etchings $(\mathrm{pH} \leq 1)$ infiltration of the smear layer may be ensured; however more calcium-phosphate is dissolved, increasing wetness and porosities in clinical conditions $[12,13]$. In fact, wetness may be already influenced by the clinical situation of pulpal pressure $[14,15]$ and the osmolarity of acidic primers [16], which favour the outward movement of dentinal fluids towards the surfaces of bonding.

To allow homogeneous infiltration of the smear in sound dentin, it was suggested that a thin smear layer of particles 
TABLE 1: Adhesive agents used in the study (components and ingredients by manufacturer information).

\begin{tabular}{|c|c|c|}
\hline Product & Components & Main ingredients \\
\hline \multirow{2}{*}{$\begin{array}{l}\text { Clearfil SE Bond } \\
\text { (Kuraray, Japan) }\end{array}$} & Self-etching primer & 10-MDP, HEMA, hydrophilic dimethacrylate, photoinitiator, and water \\
\hline & Bond & $\begin{array}{l}\text { 10-MDP, HEMA, Bis-GMA, hydrophobic dimethacrylate, photoinitiators, and } \\
\text { silanated colloidal silica }\end{array}$ \\
\hline \multirow{2}{*}{$\begin{array}{l}\text { Tyrian SPE/One-Step Plus } \\
\text { (Bisco, IL, USA) }\end{array}$} & Self-etching primer & $\begin{array}{l}\text { 2-Acrylamido-2-methylpropanesulfonic acid, bis(2-(methacryloyloxy)ethyl) } \\
\text { phosphate, and ethanol }\end{array}$ \\
\hline & Bond & Biphenyl dimethacrylate, hydroxyethyl methacrylate, acetone, and glass frit \\
\hline
\end{tabular}

be produced in the surface by refining cavities with fine grit diamond burs [17]. However, this clinical procedure has not been evaluated in CAD, in which the morphology and chemical composition of the smear are different from those of unaltered dentin because of the diverse mineral/organic composition. The smear layer in CAD is richer in organic components and appears thicker than in sound dentin. The collagen component is a highly disorganized trapping mineral and cannot be easily removed even when strong acids are used [18]. A greater amount of "collagen smear layer," compared with sound dentin, may be present on the surface of CAD because acids only solubilize the mineral component of the smear layer [12]. This collagen smear layer is impermeable to the monomers which inhibits uniform infiltration and will affect the quality of the bonding. Thus, the CAD smear layer may interfere with the infiltration of self-etching adhesives regardless of the manner in which it is created.

Although many studies have investigated the influence of dentin characteristics on bonding, little research has attempted to correlate self-etchings with the heterogeneous aspects of vital CAD in daily practice under clinical conditions. By carrying out clinical studies it is possible to evaluate the morphological expression of the bonding procedure as a result of the simultaneous interaction of tooth-adhesives in clinical conditions.

The object of this study was to evaluate the characteristics of interdiffusion of two types of two-step self-etching systems with differing $\mathrm{pH}$ values, bonded clinically in CAD cavities covered with a homogeneous smear layer of debris. The osmolarity of the primer components was also calculated.

\section{Methods and Materials}

In this study we used two types of two-step self-etching systems:

(i) Clearfil SE Bond (CSE) (Kuraray, Osaka, Japan),

(ii) Tyrian SPE/One-Step Plus (TOS) (Bisco, IL, USA).

Table 1 shows the ingredients and $\mathrm{pH}$ of the adhesives tested.

2.1. Experimental Design. This study was performed under a protocol approved by the Ethical Committee at the University of Sassari, Italy.

Fifteen subjects (mean age $47 \pm 3$ years), with a total of 21 carious premolars scheduled for extraction for periodontal reasons, were included in the study. The nature and scope of the study were explained to the participants, and informed

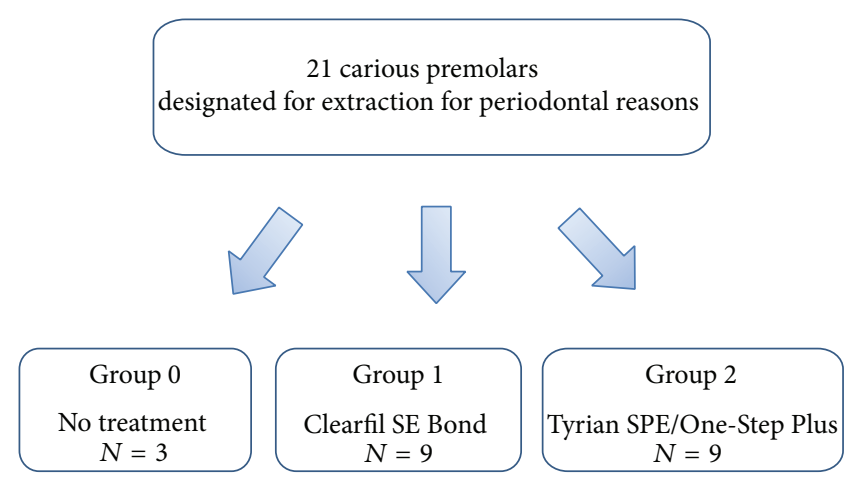

FIGURE 1: Flowchart of study methodology.

consent was obtained. The participants consented to the restoration of the carious teeth prior to extraction. Local anaesthetic without vasoconstrictor was administered to each participant and a rubber dam placed.

In the carious premolar teeth, cavities were performed by the same operator using the same equipment for both groups.

The soft caries-infected dentin was carefully removed by means of sharp spoon excavators and number 6 low speed round burs under water spray until the hard, discolored $\mathrm{CAD}$ was reached. Then, the cavities were finished using a fine diamond bur (number 8882.314.014 Komet, Lemgo, Germany) in order to create a homogeneous layer of smear debris.

After that, teeth were allocated to three groups (Figure 1), one of which $(N=3)$ received no treatment and served as a control of the created smear layer (Group 0); the other two $(N=9)$ received treatment using CSE bond (Group 1) and TOS (Group 2).

Both of the self-etching approaches were carried out according to the manufacturers' instructions (Table 2). The cavities were then bulk-filled with a flowable resin-based composite (Enamel Flow, Micerium, Avegno, Genova, Italy). Extractions were performed about 20 minutes after the polymerization of the adhesive materials.

Adhesive systems and the resin-based composite were cured using a halogen curing light (Optilux 501, Kerr Corporation, Orange, CA, USA; $11 \mathrm{~mm}$ exit window) under the standard curing mode (output wavelength range: 400-505 nm; output irradiance: $580-700 \mathrm{~mW} / \mathrm{cm}^{2}$ ) at the standardized distance of $5 \mathrm{~mm}$ from the bonding surface.

Immediately after extraction teeth were prepared for TEM processing [19]. Specimens were rinsed in distilled 
TABLE 2: pH values and application mode of the adhesive agents used in the study by manufacturer information.

\begin{tabular}{|c|c|c|c|}
\hline Product & Adhesive classification & $\mathrm{pH}$ & Mode of application \\
\hline \multirow{2}{*}{$\begin{array}{l}\text { Clearfil SE Bond } \\
\text { (Kuraray, Japan) }\end{array}$} & \multirow{2}{*}{ Mild two-step self-etching system } & \multirow{2}{*}{2.0} & $\begin{array}{l}\text { Primer: apply primer to dentin for } 20 \text { seconds and gently } \\
\text { air-blow for } 5 \text { seconds }\end{array}$ \\
\hline & & & $\begin{array}{l}\text { Bond: apply adhesive in a thin layer for } 10 \text { seconds, gently } \\
\text { air-blow, and light-cure for } 10 \text { seconds }\end{array}$ \\
\hline \multirow{2}{*}{$\begin{array}{l}\text { Tyrian SPE/One-Step Plus } \\
\text { (Bisco, IL, USA) }\end{array}$} & \multirow[t]{2}{*}{ Strong two-step self-etching system } & \multirow[t]{2}{*}{0.5} & $\begin{array}{l}\text { Primer: mix 1-2 drops of Part A with 1-2 drops of Part B, } \\
\text { apply primer to dentin for } 20 \text { seconds, and use a foam pellet } \\
\text { to blot excess from preparation, leaving surface shiny }\end{array}$ \\
\hline & & & $\begin{array}{l}\text { Bond: apply } 2 \text { coats to the dentin for } 10 \text { seconds, gently } \\
\text { air-blow, and light-cure for } 10 \text { seconds }\end{array}$ \\
\hline
\end{tabular}

water at $37^{\circ} \mathrm{C}$ and fixed in a solution of $2.5 \%$ glutaraldehyde with $0.1 \mathrm{M}$ PBS buffer, $\mathrm{pH} 7.2$, for 72 hours. The specimens were then sectioned into two halves along their longitudinal axes, and each crown was sectioned from the root using a water-cooled saw (Isomet). Small rectangular fragments of tissue perpendicular to the center of each cavity were also obtained from the occlusal surface to produce $2 \times 2 \times \sim 8 \mathrm{~mm}$ sticks. Fragments were immersed in an aqueous solution of ethylenediaminetetraacetic acid (EDTA) for 36 hours. Subsequently, samples were washed in cacodylate buffer, $\mathrm{pH}$ 7.4; postfixed in 1\% osmium tetroxide; and washed twice in cacodylate buffer. They were dehydrated using ethanol in increasing concentrations (25-100\%), embedded in epoxy resin, cut into ultrathin sections $80 \mathrm{~nm}$ in thickness using a Diatome diamond knife, and stained with lead citrate and uranyl acetate. They were then placed on 50 mesh copper grids and observed under TEM (Zeiss 109 EM Turbo).

Osmolarity of each self-etching primer was performed using a microosmometer (The Advanced Micro-Osmometer, Model 3300, Advanced Instrument Inc., Norwood, Massachusetts, USA). For this purpose, $20 \mu \mathrm{L}$ of CSE and TSO primers was dosed, and osmolarity was analysed in duplicate with the microosmometer, which utilizes freezing point depression thermodynamics. Measurement of the freezing point allows concentrations to be determined with greatest precision owing to the inherent isolation of the sample from the environment by the iced blanket generated when the sample freezes. Each sample was analysed five times in a single batch along the same day.

\section{Results and Discussion}

\subsection{TEM Observations}

Group 0. A porous, homogeneous layer of debris formed by degenerated fibrils trapping residual crystallites covered the surface of CAD. Tubules within the surface appeared obstructed by dense smear plugs rich in minerals, while others below the surface of the bur-cut dentin seemed occluded by crystals of different electron densities (Figure 2).

Group 1: CSE. The hybrid layer of about $0.5 \mu \mathrm{m}$ depth exhibited different sublayers, clearly dissimilar in micromorphology and electron densities (Figure 3).

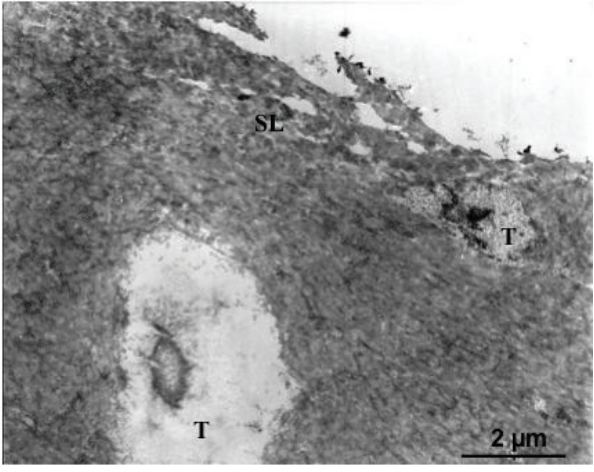

FIGURE 2: TEM photomicrographs of the control group showing the surface of affected dentin homogeneously covered by a porous layer of smear debris (SL) formed by degenerated collagen fibril and minerals. Tubules $(\mathrm{T})$ of the bur-cut dentin are smear plugged while the others below are occluded by large crystallites of different electron densities.

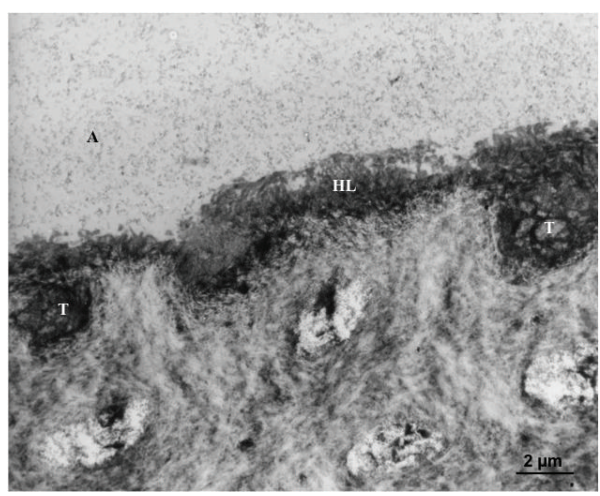

FIgURE 3: The mild self-etching Clearfil SE Bond creates a hybrid layer (HL) of about $0.1 \mathrm{um}$ depth showing different sublayers of micromorphology and electron densities that can be ascribed to the interaction of the self-etching within the features of affected dentin.

The top layer exhibited an irregular and ruffled border of retained, hybridized smear layer of denatured collagen and scattered mineralized debris. Smear layer material was also seen within the dentin front retained in areas formed by partial dissolution of the peritubular affected dentin (Figure 4). 


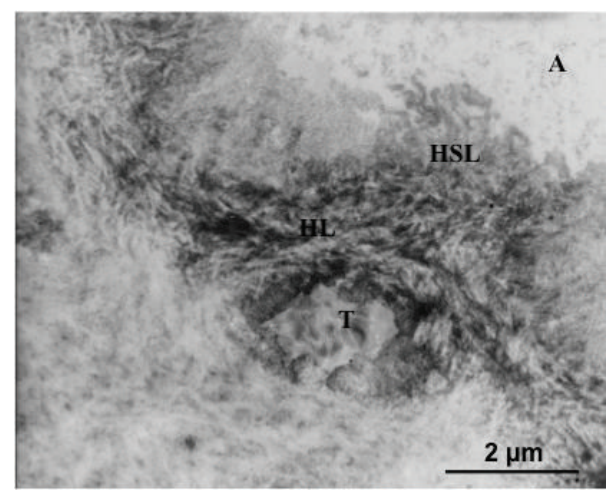

FIgURE 4: An irregular and ruffled border of hybridized smear layer residue is evident at the top of interdiffusion. The dentin front shows voids formed by the partial dissolution of the peritubular dentin, which retain hypermineralized debris.

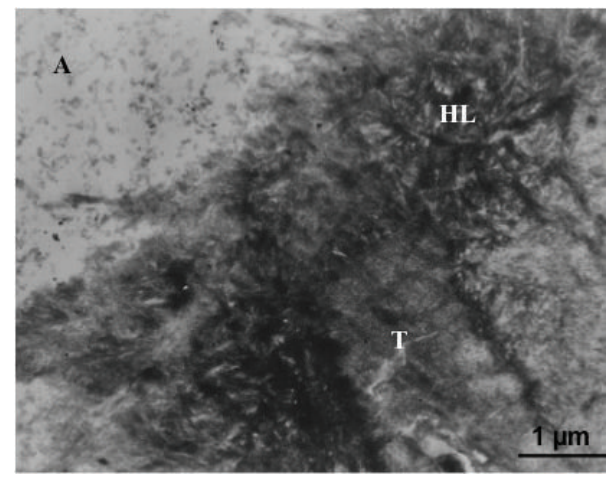

Figure 5: Infiltration of affected collagen shows dense crystallites still protecting the affected fibres within the submicron hybrid layer that is attributed to the proper mode of interaction of the mild functional monomer 10-MDP contained in Clearfil SE Bond.

Below this resin infiltered smear layer, the intertubular and peritubular affected dentin appeared tightly infiltered (Figure 5). It was possible to recognize hydroxyapatite (HAP) crystals mostly retained within the affected collagen fibrils. A reinforcement of clearly banded collagen fibrils was seen in the deepest zone of the interdiffusion making a sharply demarked reinforcement to the hybrid base.

Resin tags were not observed in the tubules which retained crystals occasionally associated with mineralized bacterial fragments. Nevertheless, tubules within the interface exhibited extreme electron refraction due to the densely infiltrated peritubular dentine, which formed a "ring-shaped" reinforcement around the orifices extended in the intertubular areas (Figure 6).

Group 2: TSO. A $0.2 \mu \mathrm{m}$ thick hybrid layer was discerned in TSO interdiffusion. No smear layer was detected but a strongly demineralized front of denatured and ragged fibrils formed the uppermost infiltered layer. Collagen fibres, associated with detached crystals, were seen arising from the top and projected into the adhesive layer (Figure 7).

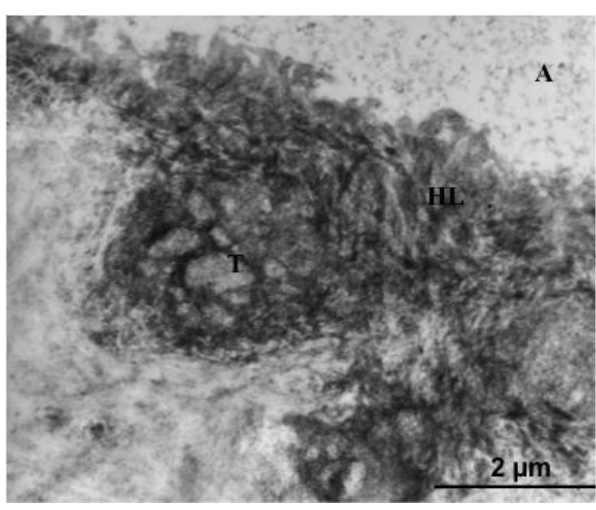

FIGURE 6: Tubules within the interface of Clearfil SE Bond exhibited extreme electron refraction due to the dense infiltration of the peritubular dentine. This hybridization generates a "ring-shaped" reinforcement extended towards the intertubular dentin, which may be interpreted as a morphological aspect of high chemical bonding.

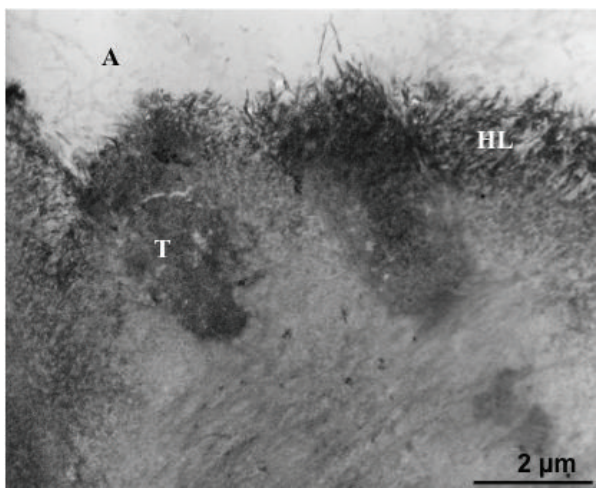

Figure 7: The strong self-etching system Tyrian SPE/One-Step Plus forms a 0.2 um thick hybrid layer (HL) completely deprived of smear layer and HAP as an effect of the modality of interaction of the low $\mathrm{pH}$ of the primer within the affected dentin surface.

Below this smear layer deprived front, the subsurface was characterized by a band of strongly demineralized collagen, separated by large interfibrillar spaces, and porosities. Electron-dense deposits were also seen probably due to reprecipitation of amorphous calcium-phosphates released by the diffusion of the acidic monomer (Figure 8).

At the base of the interdiffusion, a porous band of partially demineralized collagen was seen forming an irregular deeper layer of weak electron densities.

Resin tags were not formed in the dentinal tubules which remained obstructed by crystals. However, dissolution of peritubular dentin and porosities were observed extending toward the intertubular regions (Figure 9).

3.2. Osmolarity Analyses of the Primers. Figure 10 shows that the osmolarity of CSE primer was $3220 \mathrm{mOsm} / \mathrm{kg}$ and the osmolarity of TSO primer demonstrated values of $8200 \mathrm{mOsm} / \mathrm{kg}$. 


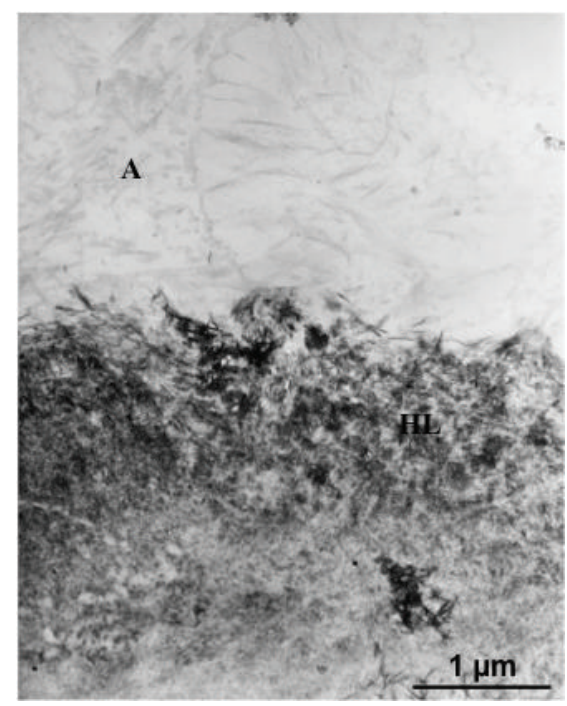

Figure 8: The top front of interdiffusion is formed by a band of hydroxyapatite deplete fibres with large interfibrillar spaces. Porosities are crossing the interdiffusion of Tyrian SPE/One-Step Plus. The pickup of the heavy metal stains which characterize the ultrathin TEM section most likely represents the dissolved calcium-phosphates which are included in the interdiffusion. Such resin-encapsulated calcium-phosphates within the exposed collagen fibrils are rather soluble and may result in instability of the interdiffusion and hydrolytic degradation over time.

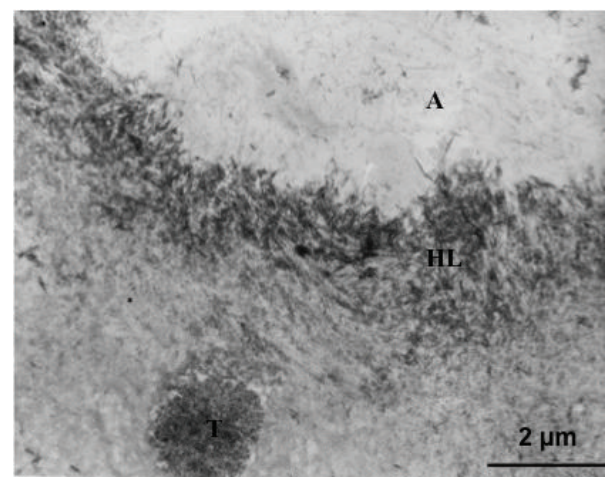

FIGURE 9: The dentinal tubules appeared obstructed by crystals, which were not affected by the low $\mathrm{pH}$ of Tyrian SPE/One-Step Plus. Also, the dissolution of peritubular dentin may be related to the acidity of the primer that was not buffered by the tubular crystals. This results in an increase of intertubular demineralization and wetness, which adversely affects the infiltration of the resins.

\section{Discussion}

Despite the important developments in adhesion in the last decades, bonding in CAD requires further understanding and improvement. Several aspects of enhancing strength and durability of CAD bonding have to be clarified, including understanding the complex heterogeneity and composition of affected dentin, an understanding of the features of the surfaces exposed after cavity preparation and the influence of the characteristics of the adhesives. This increased understanding would be helpful in obtaining a tight resin/dentin

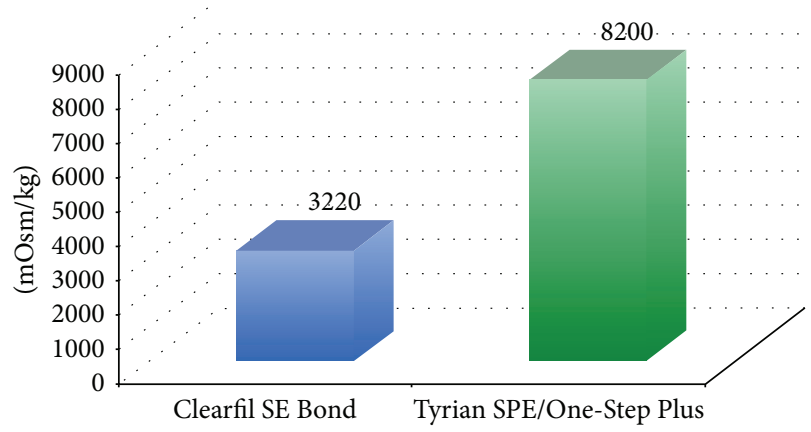

Figure 10: Mean values of the osmolarity of the tested materials.

interdiffusion, which is compatible with a stable bond as opposed to nanoleakage and hydrolysis by the host derived matrix metalloproteinase.

In this study, in order to evaluate the capacity of interaction of the two self-etchings in CAD, we standardized the smear by finishing the cavity with an extra-fine diamond bur. This resulted in a thinner layer of smear debris, allowing infiltration of the affected dentin regardless of the $\mathrm{pH}$ of the acidic monomers [17]. At the same time, we studied the TEM appearance of the bonding obtained under clinical conditions of pulpal pressure, from the point of view of the $\mathrm{pH}$ and osmolarity of the adhesives used.

In the case of the CSE, mild self-etching system, the interdiffusion occurred with the inclusion of the top smear layer and the different features of CAD. Acidification occurred only partially, in comparison to the strong self-etching system TSO, keeping the demineralized HAP crystallites still attached to the fibrils.

The hybrid layer was characterized by retained smear layer residues and scattered needle shape crystallites as an effect of the mild acidity of CSE. No effects attributed to the movement of fluids were observed within the interdiffusion, which appeared tightly sealed by the adhesive resin of CSE. The prevention of movement of fluids through the interface could be attributed to the mild demineralization of intertubular affected dentin and the low osmolarity of the primer, rather than to the presence of mineral occlusion in the dentinal tubules, even in the presence of pulpal pressure. Under clinical conditions of pulpal pressure, water movement may occur by way of the channels of least resistance, that is, the intratubular minerals and the thin peritubular dentin, towards the porous intertubular collagen of CAD, producing interference in the reinforcement and voids of resin [15]. Although the mineral deposits were retained in the tubules, peritubular dentin appeared densely infiltered and hybridized toward the intertubular regions without evidence of porosities. Also, the hybridization of the peritubular dentin generated a "ring-shaped" reinforcement which has been claimed to be a source of calcium ions that strongly contribute to the high chemical bonding of self-etchings [20].

The high presence of HAP, still protecting the affected fibres within the submicron hybrid layer, is attributed to the proper mode of interaction of the mild functional monomer 10-MDP in CSE. The 10-MDP has the capacity to interact 
with the residual HAP establishing primary ionic binding $[11,15,21-24]$. This chemical interaction causes the formation of a regularly layered structure on the surface, within which highly insoluble calcium salts are deposited. Also, the TEM observations show that CLE adequately infiltrates HAP and the partially demineralized collagen matrix, protecting fibrils from being exposed and degraded in environmental fluids [24-27].

The strong self-etching system exhibited different characteristics in CAD. The low $\mathrm{pH}$ and high osmolarity of TSO primer played an important role in the morphological aspect of this hybrid layer.

The dentinal front was characterized by deep dissolution of the smear layer and HAP crystallites due to the effect of the strong $\mathrm{pH}$ on the affected surface. This produced a hybrid layer whose formation might be similar to that created by the etch and rinse adhesives [28]. Within the calciumdepleted surface the monomer infiltration occurred due to a primary diffusion-based mechanism, creating a bond which is thought to be lower in bond strength than the chemical bonded hybrid layer [22]. The strong pickup of the heavy metal stains which characterized the TEM photomicrographs of this strongly demineralized hybrid layer most likely represents dissolved calcium-phosphates. Such resin-encapsulated calcium-phosphates within the exposed collagen fibrils are somewhat soluble $[22,29]$ and may result in instability of the interdiffusion, with hydrolytic degradation over time [30].

Also the acidity of TSO and the lower buffer capacity of the tubular crystallites may explain the dissolution of peritubular dentin $[12,13]$. These factors resulted in an increase of intertubular demineralization. As a result, demineralization channels were observed extending from both peritubular and intertubular areas and they consisted of small, close-knit aqueous porosities forming a chelation slot, low in infiltrated monomers.

The strong demineralization and the high osmolarity of the primer may have also caused movement of dentinal fluids toward the surface explaining the observation of ragged fibres and detached dentin chips within the adhesive front. Another explanation of the evidence of fluid movements may be the high content of water in self-etching. TSO contains a high concentration of water, which is required to dissociate acidic monomers into ionized forms able to permeate the surface. Water content increases as the acidity of the primer lowers [31]. Mixtures with a high concentration of water and ethanol, such as TSO, may impair polymerization of the monomers within the infiltered surface [32] allowing the passage of fluids. Furthermore, TSO uses a solvent-rich, hydrophilic adhesive coating rather than a hydrophobic one, such as in CLE. This hydrophilic adhesive was not able to prevent the formation of a permeable membrane after the polymerization, explaining the evidence of water movement within the interface and water porosity in the hybrid layer.

Moreover, the high concentration of solvent in TSO may have an influence on the low bonding demonstrated in clinical studies over 18 months of oral exposure [31]. The low effectiveness of TSO has been explained by an incomplete evaporation of the solvent within the interface, preventing an adequate monomer conversion, which may affect the mechanical properties, bond strength, and retention rates of the bonding when in an oral environment.

The poor infiltration related to the overaggressive effect of the demineralization, together with the presence of a hydrophilic coating and an incomplete evaporation of the solvent in TSO, may cause a passage of fluids within the hybrid layer, which leads to the degradation of the interdiffusion of TSO in CAD over time.

\section{Conclusions}

Under clinical conditions, the effectiveness of bonding in CAD with a homogeneous smear layer is strongly influenced by the chemistry of self-etchings.

The mild CSE self-etching produces a dense and homogeneous infiltration of the CAD smear layer, the peritubular dentin, and the affected collagen fibrils. The presence of HAP in the fibrils seems to ensure a tight bonding which is able to resist degradation over time.

Conversely, the strong TSO completely dissolved the smear layer, producing, at the same time, a poor infiltration of the CAD interface. This poor infiltration may be related to the overaggressive effect of the demineralization that, together with a hydrophilic coating and the incomplete evaporation of the solvent in TSO, may be the cause of a passage of fluids within the hybrid layer.

Far from helping the homogeneity of interdiffusion, increasing the $\mathrm{pH}$ and osmolarity of self-etchings in CAD may cause nonhomogeneous reinforcement with infiltration of oral fluids and the failure of the bonding over time.

\section{Conflict of Interests}

The authors declare that there is no conflict of interests regarding the publication of this paper.

\section{References}

[1] M. Degrange and J. F. Roulet, Minimally Invasive Restorations with Bonding, Quintessence, Chicago, Ill, USA, 1997.

[2] M. Nakajima, H. Sano, M. F. Burrow et al., “Tensile bond strength and SEM evaluation of caries-affected dentin using dentin adhesives," Journal of dental research, vol. 74, no. 10, pp. 1679-1688, 1995.

[3] K. Ogawa, Y. Yamashita, T. Ichijo, and T. Fusayama, "The ultrastructure and hardness of the transparent layer of human carious dentin," Journal of Dental Research, vol. 62, no. 1, pp. 7$10,1983$.

[4] G. W. Marshall, S. Habelitz, R. Gallagher, M. Balooch, G. Balooch, and S. J. Marshall, "Nanomechanical properties of hydrated carious human dentin," Journal of Dental Research, vol. 80, no. 8, pp. 1768-1771, 2001.

[5] M. Nakajima, H. Sano, M. F. Burrow et al., "Tensile bond strength and SEM evaluation of caries-affected dentin using dentin adhesives," Journal of Dental Research, vol. 74, no. 10, pp. 1679-1688, 1995.

[6] S. Ito, T. Saito, F. R. Tay, R. M. Carvalho, M. Yoshiyama, and D. H. Pashley, "Water content and apparent stiffness of non-caries versus caries-affected human dentin," Journal of Biomedical 
Materials Research Part B: Applied Biomaterials, vol. 72, no. 1, pp. 109-116, 2005.

[7] Y. Wang, P. Spencer, and M. P. Walker, "Chemical profile of adhesive/caries-affected dentin interfaces using Raman microspectroscopy," Journal of Biomedical Materials Research Part A, vol. 81, no. 2, pp. 279-286, 2007.

[8] C. Kaaden, J. M. Powers, K.-H. Friedl, and G. Schmalz, "Bond strength of self-etching adhesives to dental hard tissues," Clinical Oral Investigations, vol. 6, no. 3, pp. 155-160, 2002.

[9] M. Toledano, R. Osorio, L. Ceballos et al., "Microtensile bond strength of several adhesive systems to different dentin depths," American Journal of Dentistry, vol. 16, no. 5, pp. 292-298, 2003.

[10] D. H. Pashley, B. Ciucchi, H. Sano, and J. A. Horner, "Permeability of dentin to adhesive agents," Quintessence International, vol. 24, no. 9, pp. 618-631, 1993.

[11] A. Santini, V. Ivanovic, R. Ibbetson, and E. Milia, "Influence of marginal bevels on microleakage around Class $\mathrm{V}$ cavities bonded with seven self-etching agents," American Journal of Dentistry, vol. 17, no. 4, pp. 257-261, 2004.

[12] D. H. Pashley and R. M. Carvalho, "Dentine permeability and dentine adhesion," Journal of Dentistry, vol. 25, no. 5, pp. 355372, 1997.

[13] S.-M. Kwong, F. R. Tay, H.-K. Yip, L.-H. Kei, and D. H. Pashley, "An ultrastructural study of the application of dentine adhesives to acid-conditioned sclerotic dentine," Journal of Dentistry, vol. 28, no. 7, pp. 515-528, 2000.

[14] S. Sauro, D. H. Pashley, M. Montanari et al., "Effect of simulated pulpal pressure on dentin permeability and adhesion of selfetch adhesives," Dental Materials, vol. 23, no. 6, pp. 705-713, 2007.

[15] E. Milia, R. Pinna, G. Castelli et al., “TEM morphological characterization of a one-step self-etching system applied clinically to human caries-affected dentin and deep sound dentin," American Journal of Dentistry, vol. 25, no. 6, pp. 321-326, 2012.

[16] D. H. Pashley, "Smear layer: overview of structure and function," Proceedings of the Finnish Dental Society, vol. 88, pp. 215-224, 1992.

[17] R. B. Ermis, J. de Munck, M. V. Cardoso et al., "Bond strength of self-etch adhesives to dentin prepared with three different diamond burs," Dental Materials, vol. 24, no. 7, pp. 978-985, 2008.

[18] P. Spencer, Y. Wang, M. P. Walker, and J. R. Swafford, "Molecular structure of acid-etched dentin smear layers-in situ study," Journal of Dental Research, vol. 80, no. 9, pp. 1802-1807, 2001.

[19] E. Milia, M. R. Lallai, and F. García-Godoy, "In vivo effect of a self-etching primer on dentin," American Journal of Dentistry, vol. 12, no. 4, pp. 167-171, 1999.

[20] E. Milia, E. Cumbo, R. J. A. Cardoso, and G. Gallina, "Current dental adhesives systems. A narrative review," Current Pharmaceutical Design, vol. 18, no. 34, pp. 5542-5552, 2012.

[21] M. C. G. Erhardt, M. Toledano, R. Osorio, and L. A. Pimenta, "Histomorphologic characterization and bond strength evaluation of caries-affected dentin/resin interfaces: effects of longterm water exposure," Dental Materials, vol. 24, no. 6, pp. 786798, 2008.

[22] Y. Yoshida, K. Nagakane, R. Fukuda et al., "Comparative study on adhesive performance of functional monomers," Journal of Dental Research, vol. 83, no. 6, pp. 454-458, 2004.

[23] M. Yoshiyama, A. Urayama, T. Kimochi, T. Matsuo, and D. H. Pashley, "Comparison of conventional vs. self-etching adhesive bonds to caries-affected dentin," Operative Dentistry, vol. 25, no. 3, pp. 163-169, 2002.
[24] S. Inoue, K. Koshiro, Y. Yoshida et al., "Hydrolytic stability of self-etch adhesives bonded to dentin," Journal of Dental Research, vol. 84, no. 12, pp. 1160-1164, 2005.

[25] U. Salz, J. Zimmermann, F. Zeuner, and N. Moszner, "Hydrolytic stability of self-etching adhesive systems," Journal of Adhesive Dentistry, vol. 7, no. 2, pp. 107-116, 2005.

[26] P. Farge, L. Alderete, and S. M. M. Ramos, "Dentin wetting by three adhesive systems: influence of etching time, temperature and relative humidity," Journal of Dentistry, vol. 38, no. 9, pp. 698-706, 2010.

[27] E. Milia, G. Castelli, A. Bortone et al., "Short-term response of three resin-based materials as desensitizing agents under oral environmental exposure," Acta Odontologica Scandinavica, vol. 71, no. 3-4, pp. 599-609, 2013.

[28] E. Milia and A. Santini, "Ultrastructural transmission electron microscopy (TEM) study of hybrid layers formed beneath a one-bottle adhesive system using the total-etch technique and a self-etching system," Quintessence International, vol. 34, no. 6, pp. 447-452, 2003.

[29] B. van Meerbeek, K. Yoshihara, Y. Yoshida, A. Mine, J. de Munck, and K. L. van Landuyt, "State of the art of self-etch adhesives," Dental Materials, vol. 27, no. 1, pp. 17-28, 2011.

[30] W. W. Brackett, D. A. Covey, and H. A. St. Germain Jr., "Oneyear clinical performance of a self-etching adhesive in class $\mathrm{V}$ resin composites cured by two methods," Operative Dentistry, vol. 27, no. 3, pp. 218-222, 2002.

[31] A. D. Loguercio and A. Reis, "Application of a dental adhesive using the self-etch and etch-and-rinse approaches: an 18month clinical evaluation," The Journal of the American Dental Association, vol. 139, no. 1, pp. 53-61, 2008.

[32] E. L. Pashley, Y. Zhang, P. E. Lockwood, F. A. Rueggeberg, and D. H. Pashley, "Effects of HEMA on water evaporation from waterHEMA mixtures," Dental Materials, vol. 14, no. 1, pp. 6-10, 1998. 

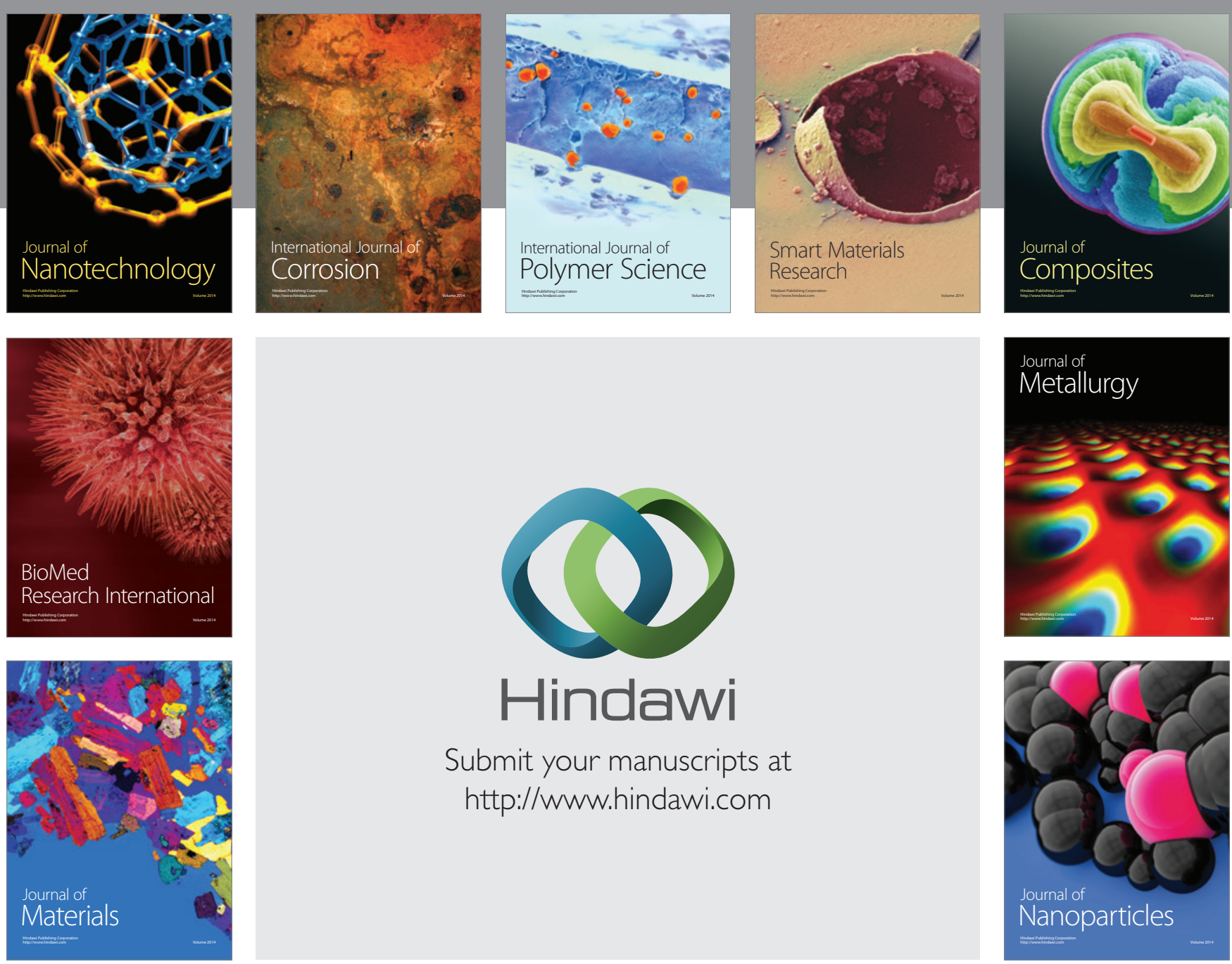

Submit your manuscripts at http://www.hindawi.com
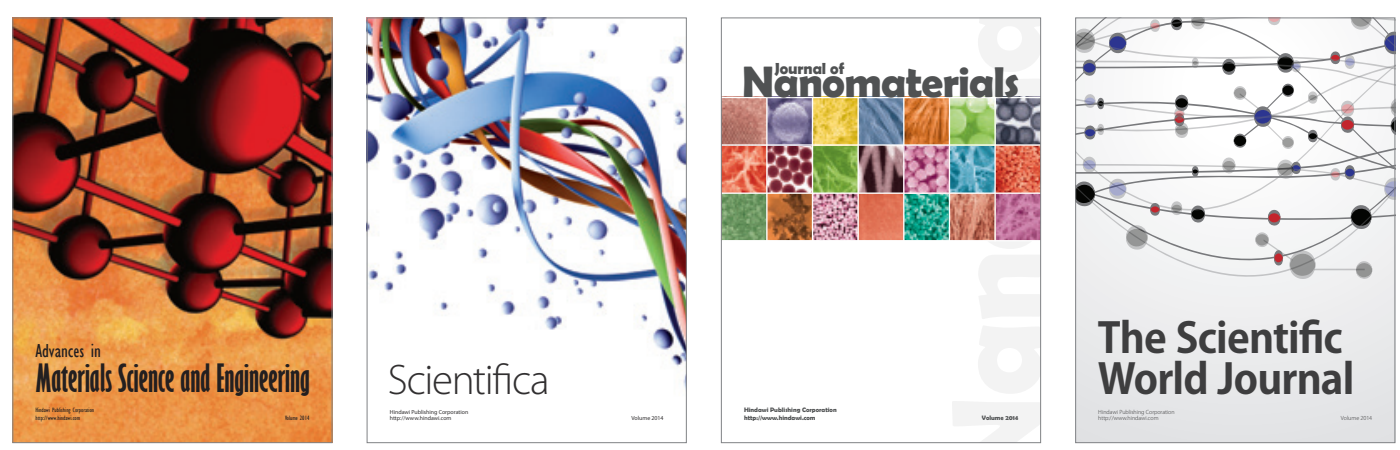

\section{The Scientific World Journal}
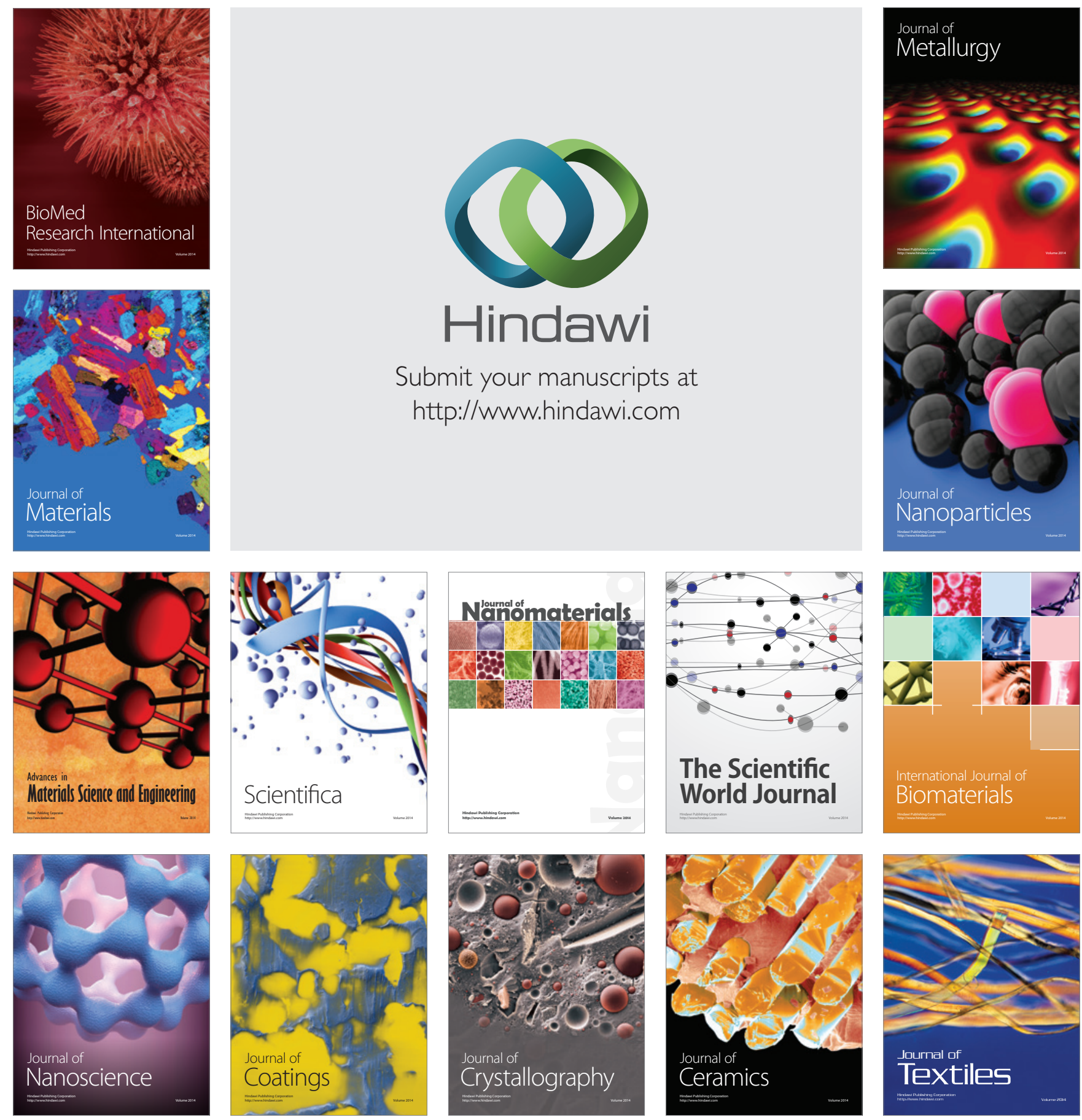